\title{
High-energy breakfast with low-energy dinner decreases overall daily hyperglycaemia in type 2 diabetic patients: a randomised clinical trial
}

\author{
Daniela Jakubowicz • Julio Wainstein • Bo Ahrén • \\ Yosefa Bar-Dayan • Zohar Landau • \\ Hadas R. Rabinovitz • Oren Froy
}

Received: 23 November 2014 / Accepted: 28 January 2015/Published online: 1 March 2015

(C) Springer-Verlag Berlin Heidelberg 2015

\begin{abstract}
Aims/hypothesis High-energy breakfast and reduced-energy dinner (Bdiet) significantly reduces postprandial glycaemia in obese non-diabetic individuals. Our objective was to test whether this meal schedule reduces postprandial hyperglycaemia (PPHG) in patients with type 2 diabetes by enhancing incretin and insulin levels when compared with high-energy dinner and reduced-energy breakfast (Ddiet).

Methods In a randomised, open label, crossover design performed in a clinic setting, 18 individuals (aged 30-70 years with BMI $\left.22-35 \mathrm{~kg} / \mathrm{m}^{2}\right)$ with type 2 diabetes $(<10$ years duration) treated with metformin and/or diet were given either Bdiet or Ddiet for 7 days. Participants were randomised by a person not involved in the study using a coin flip. Postprandial levels of plasma glucose, insulin, C-peptide and intact and total glucagon-like peptide-1 (iGLP-1 and tGLP-1) were assessed. The Bdiet included 2,946 kJ breakfast, 2,523 kJ lunch and $858 \mathrm{~kJ}$ dinner. The Ddiet comprised $858 \mathrm{~kJ}$ breakfast, $2,523 \mathrm{~kJ}$ lunch and 2,946 kJ dinner.

Results Twenty-two individuals were randomised and 18 analysed. The AUC for glucose ( $\left.\mathrm{AUC}_{\text {glucose }}\right)$ throughout the day was $20 \%$ lower, whereas $\mathrm{AUC}_{\text {insulin, }} \mathrm{AUC}_{\mathrm{C} \text {-peptide }}$ and
\end{abstract}

D. Jakubowicz $(\varangle) \cdot J$. Wainstein $\cdot$ Y. Bar-Dayan $\cdot$ Z. Landau Diabetes Unit, E. Wolfson Medical Center, Sackler Faculty of Medicine, Tel Aviv University, Holon 58100, Israel

e-mail: daniela.jak@gmail.com

B. Ahrén

Department of Clinical Sciences, Faculty of Medicine, Lund

University, Lund, Sweden

H. R. Rabinovitz $\cdot$ O. Froy $(\square)$

Institute of Biochemistry, Food Science and Nutrition, The Robert $\mathrm{H}$.

Smith Faculty of Agriculture, Food and Environment, The Hebrew

University of Jerusalem, Rehovot 76100, Israel

e-mail: oren.froy@mail.huji.ac.il
$\mathrm{AUC}_{\mathrm{tGLP}-1}$ were $20 \%$ higher for the Bdiet than the Ddiet. Glucose $\mathrm{AUC}_{0-180 \min }$ and its peak were both lower by $24 \%$, whereas insulin $\mathrm{AUC}_{0-180 \mathrm{~min}}$ was $11 \%$ higher after the Bdiet than the Ddiet. This was accompanied by $30 \%$ higher tGLP-1 and $16 \%$ higher iGLP-1 levels. Despite the diets being isoenergetic, lunch resulted in lower glucose (by 21-25\%) and higher insulin (by 23\%) with the Bdiet vs Ddiet.

Conclusions/interpretation High energy intake at breakfast is associated with significant reduction in overall PPHG in diabetic patients over the entire day. This dietary adjustment may have a therapeutic advantage for the achievement of optimal metabolic control and may have the potential for being preventive for cardiovascular and other complications of type 2 diabetes.

Trial registration ClinicalTrials.gov NCT01977833

Funding No specific funding was received for the study.

Keywords Breakfast · Clock · Diabetes · Dinner - GLP-1 · Insulin · Timing
Abbreviations
Bdiet High-energy breakfast and reduced-energy dinner
Ddiet High-energy dinner and reduced-energy breakfast
iGLP-1 Intact glucagon-like peptide-1
PPHG Postprandial hyperglycaemia
tGLP-1 Total glucagon-like peptide-1

\section{Introduction}

Postprandial hyperglycaemia (PPHG) contributes $31-70 \%$ to $\mathrm{HbA}_{1 \mathrm{c}}$ values [1] and is strongly associated with increased cardiovascular risk in type 2 diabetes [2,3]. Therefore, postprandial glycaemia is an important treatment target in type 2 
diabetes. Post-meal glycaemia displays a clear circadian pattern with a more prolonged and higher response to an identical meal in the evening vs the morning [4-8]. This is achieved in part by the circadian secretion and activity of enzymes and hormones involved in the regulation of postprandial glycaemia [9-13]. Indeed, the circadian clock controls glucagon-like peptide 1 (GLP-1) secretion in intestinal L cells [9], insulin secretion in beta cells [14, 15], hepatic insulin extraction [7] and insulin-dependent glucose transporter GLUT 4 expression in skeletal muscle [10, 13].

While the master clock is entrained to the light-dark cycles [16], meal timing and feeding schedules exert strong entraining effects on peripheral oscillators maintaining the internal synchrony of most metabolic processes $[17,18]$. Studies in animals and humans have shown that altered meal timing, such as skipping breakfast or high energy intake at dinner, are associated with disrupted clock gene expression, increased lipogenesis, higher $\mathrm{HbA}_{1 \mathrm{c}}$ and poor glycaemic control [4, 19-22]. In contrast, high-energy breakfast with no dinner or time-restricted feeding reversed the impaired clock gene expression, resulting in decreased plasma glucose and triacylglycerol levels and reduced body weight in animal models of obesity and type 2 diabetes [12, 22, 23]. The different effects of meals during the day may therefore be modulated by different energy loads to achieve optimal glucose homeostasis. However, the effect of different meal timing schedules on overall incretin, insulin and glycaemic excursion during the entire day has not been previously explored in individuals with type 2 diabetes. In this study, we assessed the differential influence of two isoenergetic diets with different meal timing schedules on PPHG over the entire day and the corresponding changes in insulin and incretin hormones in patients with type 2 diabetes. The two dietary schedules were as follows: one with high-energy breakfast and lower-energy dinner (Bdiet) and the other with high-energy dinner and lower-energy breakfast (Ddiet).

\section{Methods}

Participants The study population initially included 22 individuals who had type 2 diabetes for less than 10 years and an $\mathrm{HbA}_{1 \mathrm{c}}$ level of $7-9 \%(53-75 \mathrm{mmol} / \mathrm{mol})$ on recruitment (Table 1). Individuals aged $30-70$ years with BMI $22-35 \mathrm{~kg} / \mathrm{m}^{2}$ were included. None of the participants had impaired thyroid, renal or liver function, pulmonary disease, psychiatric, immunological or neoplastic diseases or severe diabetic complications, such as cardiovascular disease, cerebrovascular disease, proliferative diabetic retinopathy or gastroparesis and none had undergone bariatric surgery. All participants were insulin-naive and patients taking oral hypoglycaemic agents other than metformin were excluded. Those on GLP-1 analogues or anorectic drugs or those on steroid treatment were not allowed to participate. The
Table 1 Clinical and anthropometric characteristics

\begin{tabular}{ll}
\hline Characteristic & \\
\hline No. of patients & 18 \\
Sex (no. male/no. female) & $8 / 10$ \\
Age (years) & $57.8 \pm 4.7$ \\
Weight $(\mathrm{kg})$ & $76.8 \pm 13.5$ \\
BMI $\left(\mathrm{kg} / \mathrm{m}^{2}\right.$ ) & $28.1 \pm 2.9$ \\
Waist circumference $(\mathrm{cm})$ & $94 \pm 5.8$ \\
Systolic BP (mmHg) & $130.7 \pm 7.2$ \\
Diastolic BP (mmHg) & $80.6 \pm 7.46$ \\
HbA 1 ( $\%)$ & $7.6 \pm 0.4$ \\
HbA ${ }_{1 \mathrm{c}}(\mathrm{mmol} / \mathrm{mol})$ & $59 \pm 2$ \\
Time since diagnosis of diabetes (years) & $9.3 \pm 5$ \\
Fasting blood glucose (mmol/l) & $7.2 \pm 0.5$ \\
Fasting plasma insulin (pmol/l) & $49.3 \pm 9$ \\
\hline
\end{tabular}

Data are shown as $n$ or as mean $\pm \mathrm{SE}$

Helsinki Committee of the Wolfson Medical Center in Holon, Israel approved the study. All the participants gave their informed consent. The study was registered at ClinicalTrials.gov (NCT01977833).

Study design This was a randomised, open-label, crossoverwithin-subject clinical trial. Participants were randomised by a person not involved in the study using a coin flip. We performed two separate testing days each over the course of $14 \mathrm{~h}$. Test meals were provided in the clinic as breakfast, lunch and dinner with energy and composition according to the assigned diet schedule (Table 2). The participants consumed their meals within $15 \mathrm{~min}$, with breakfast at 08:00 hours, lunch at 13:00 hours and dinner at 19:00 hours. The diet during both testing days provided a total daily energy intake of $6,276 \pm$ $105 \mathrm{~kJ}$ ( $23 \%$ fat, $46 \%$ carbohydrates, $31 \%$ protein) with identical macronutrient content and composition, but different meal timing distribution. The two meal timing schedules were either breakfast diet (Bdiet) or dinner diet (Ddiet). The Bdiet consisted of a large breakfast $(2,946 \mathrm{~kJ} ; 22 \%$ fat, $47 \%$ carbohydrates, $31 \%$ protein), a medium-sized lunch $(2,523 \mathrm{~kJ} ; 23 \%$ fat, $50 \%$ carbohydrates, $27 \%$ protein) and a small dinner ( $858 \mathrm{~kJ} ; 30 \%$ fat, $27 \%$ carbohydrates, $43 \%$ protein). This was reversed in the Ddiet (858 kJ breakfast, 2,523 kJ lunch and 2,946 kJ dinner). Participants followed the Bdiet and Ddiet meal plans for 6 days at home prior to the intervention day to prevent the pre-study meal pattern from influencing the results. Participants were also asked to avoid alcohol and excessive physical activity 6 days before each test day. Participants ingested their last oral therapy $24 \mathrm{~h}$ before the test day. Following a washout period of 2 weeks, the same procedure was repeated on the second test day, with each participant now crossed over to the opposite diet schedule. 
Table 2 Diet composition on the meal test days

\begin{tabular}{|c|c|c|c|c|c|}
\hline \multirow[t]{2}{*}{ Meal type } & \multirow[t]{2}{*}{ Energy content $(\mathrm{kJ})$} & \multicolumn{4}{|c|}{ Composition } \\
\hline & & Fat $(\mathrm{g})$ & Carbohydrate (g) & Protein $(g)$ & Fibre $(\mathrm{g})$ \\
\hline \multicolumn{6}{|l|}{ Large meal } \\
\hline Whole-wheat bread: two slices & 569 & 2.4 & 25.8 & 5.4 & 3.9 \\
\hline Caffè latte with non-fat milk: one tall & 418 & 0.0 & 15.0 & 10.0 & 0.0 \\
\hline Tuna, light in water: $115 \mathrm{~g}$ & 502 & 1.0 & 1.0 & 26.0 & 0.4 \\
\hline Scrambled egg: one egg & 159 & 1.5 & 0.5 & 5.5 & 0.0 \\
\hline Olive oil: two teaspoons & 335 & 9.0 & 0.0 & 0.0 & 0.0 \\
\hline Yogurt and cereal: one container & 460 & 2.0 & 20.0 & 5.0 & 3.0 \\
\hline Granola bar, dark chocolate: one bar & 502 & 2.0 & 24.0 & 5.0 & 4.0 \\
\hline Total & 2,946 & 17.9 & 86.3 & 56.9 & 11.3 \\
\hline Percentage of energy & & 22 & 47 & 31 & - \\
\hline \multicolumn{6}{|l|}{ Lunch } \\
\hline Apple: one apple & 339 & 0.5 & 21.0 & 0.3 & 3.7 \\
\hline Roasted chicken breast: $115 \mathrm{~g}$ & 782 & 4.0 & 0.0 & 35.0 & 0.0 \\
\hline Diet drink: $236 \mathrm{ml}$ & 0 & 0.0 & 0.0 & 0.0 & 0.0 \\
\hline Salad, tossed greens: one salad & 42 & 0.0 & 2.0 & 0.0 & 0.5 \\
\hline Vegetable soup: one cup & 418 & 2.0 & 20.0 & 4.0 & 4.0 \\
\hline Olive oil: two teaspoons & 335 & 9.0 & 0.0 & 0.0 & 0.0 \\
\hline Baked potato: one potato & 607 & 0.2 & 33.6 & 3.1 & 2.3 \\
\hline Total & 2,523 & 15.7 & 76.6 & 42.4 & 10.5 \\
\hline Percentage of energy & & 23 & 50 & 27 & - \\
\hline \multicolumn{6}{|l|}{ Small meal } \\
\hline Caffè Americano: one large & 63 & 0.0 & 3.0 & 1.0 & 0.0 \\
\hline Salad, fresh tomato and mozzarella: one order & 469 & 7.0 & 6.0 & 6.2 & 1.0 \\
\hline Salad, tossed greens: one salad & 42 & 0.0 & 2.0 & 0.0 & 0.5 \\
\hline Turkey breast: three slices & 251 & 0.0 & 1.0 & 14.0 & - \\
\hline Salad, mixed greens: one cup & 33 & 0.0 & 2.0 & 1.0 & 1.0 \\
\hline Total & 858 & 7.0 & 14.0 & 22.2 & 2.5 \\
\hline Percentage of energy & & 30 & 27 & 43 & - \\
\hline
\end{tabular}

Meal challenges On the day of the meal challenge, each participant reported to the laboratory at 07:00 hours after an overnight fast. Participants were asked not to take glucoselowering medication during the test days and were instructed to keep dietary logs. A dietitian reviewed these logs on the day of the meal tests and only those who had $80 \%$ adherence to the diet participated in the meal tests. Anthropometric data were collected in the morning and each group consumed their assigned meal plan: breakfast at 08:00 hours, lunch at 13:00 hours and dinner at 19:00 hours. At 07:30 hours, a catheter was placed in the antecubital vein of the nondominant arm and remained in place until 22:00 hours. Venous blood samples were collected just before breakfast $(t=0 \mathrm{~min})$ and at 15, 30, 60, 90, 120, 150 and $180 \mathrm{~min}$ after eating commenced. Blood sampling was repeated at the same time points after lunch and dinner. The primary outcome was to assess the overall postprandial GLP-1 levels in both meal schedules. The secondary outcome was to assess the overall postprandial glycaemia. Other outcomes were to assess the overall postprandial response of plasma insulin and C-peptide in both meal schedules.

Biochemical and hormonal blood analyses Plasma glucose was immediately analysed on an Olympus AU 2700 analyser (Beckman Coulter, Brea, CA, USA). Serum and plasma EDTA tubes for insulin and C-peptide were left on ice to rest for approximately $30 \mathrm{~min}$. Blood samples for determining iGLP-1 and tGLP-1 were collected into chilled tubes containing EDTA, aprotinin and diprotin A $(0.1 \mathrm{mmol} / \mathrm{l})$. Samples were centrifuged immediately at $2,000 \mathrm{~g}$ at $4^{\circ} \mathrm{C}$ for $10 \mathrm{~min}$ and stored at $-80^{\circ} \mathrm{C}$. Insulin and $\mathrm{C}$-peptide were determined by electrochemiluminescence using a Cobas 601 Roche Diagnostic analyser (Madison, WI, USA) according to the manufacturer's instructions. 
Plasma tGLP-1 and iGLP-1 were quantified using ELISA (Millipore, Billerica, MA, USA).

Sample size and power analysis Power analysis revealed that a sample size of 18 participants (18 in each treatment group; Bdiet and Ddiet) is required to provide $80 \%$ power to detect $5 \%$ difference between groups in overall postprandial plasma AUC for iGLP-1, insulin and glucose assessed after three meals in each diet intervention. To allow discontinuation during the course of the study, 22 participants were recruited.

Statistical analyses The results are expressed as mean \pm SEM. For time series, a two-way ANOVA (time $\times$ diet) was performed and a least-significant difference $t$ test post hoc analysis was used for comparison between the diets at each time point. Means comparisons were made using the CI of the estimators. AUCs were calculated using the trapezoidal rule. A Student's $t$ test for paired data was used for comparing the AUC at different time intervals. In addition, a multivariate ANOVA for repeated measurements was performed to assess between- and within-subject effects for diet and time. A $p$ value $\leq 0.05$ was considered statistically significant. Statistical analysis was performed using SPSS (version 18) software (www14.software.ibm.com/download/data/web/en US/trialprograms/W110742E06714B29.html).

\section{Results}

Participants Twenty-two individuals from the Wolfson Diabetes Unit outpatient clinic were enrolled into the study (Fig. 1). The study period from recruitment and including follow-up was December 2013 to April 2014. After completing the first all-meals testing day, four participants dropped out (two from the Bdiet group and two from the Ddiet group), because of difficulties getting to the clinic. Eighteen individuals (eight men, ten women) completed the study. These patients had an average age of $57.8 \pm 4.7$ years, controlled type 2 diabetes of $9.3 \pm 5.0$ years duration, $\mathrm{HbA}_{1 \mathrm{c}}$ values of $7.6 \pm$ $0.4 \%(59 \pm 2 \mathrm{mmol} / \mathrm{mol})$ and BMI of $28.1 \pm 2.9 \mathrm{~kg} / \mathrm{m}^{2}$ (Table 1). Eight patients were treated with diet alone, whereas ten were treated with diet and metformin. Five patients had a history of hypertension and were treated with thiazides, angiotensin-converting enzyme inhibitors and/or calcium antagonists.

Plasma glucose and hormonal profiles of Bdiet vs Ddiet group Fasting plasma glucose, insulin, C-peptide, tGLP-1 and iGLP-1 did not differ significantly between the two test days or the groups (Fig. 2). Integrated $\mathrm{AUC}_{\text {glucose }}$ after breakfast, lunch and dinner was $20 \%$ lower in the Bdiet group than in the Ddiet $(p<0.001, t$ test) group (Fig. 2). Integrated
Fig. 1 Flow diagram of recruitment

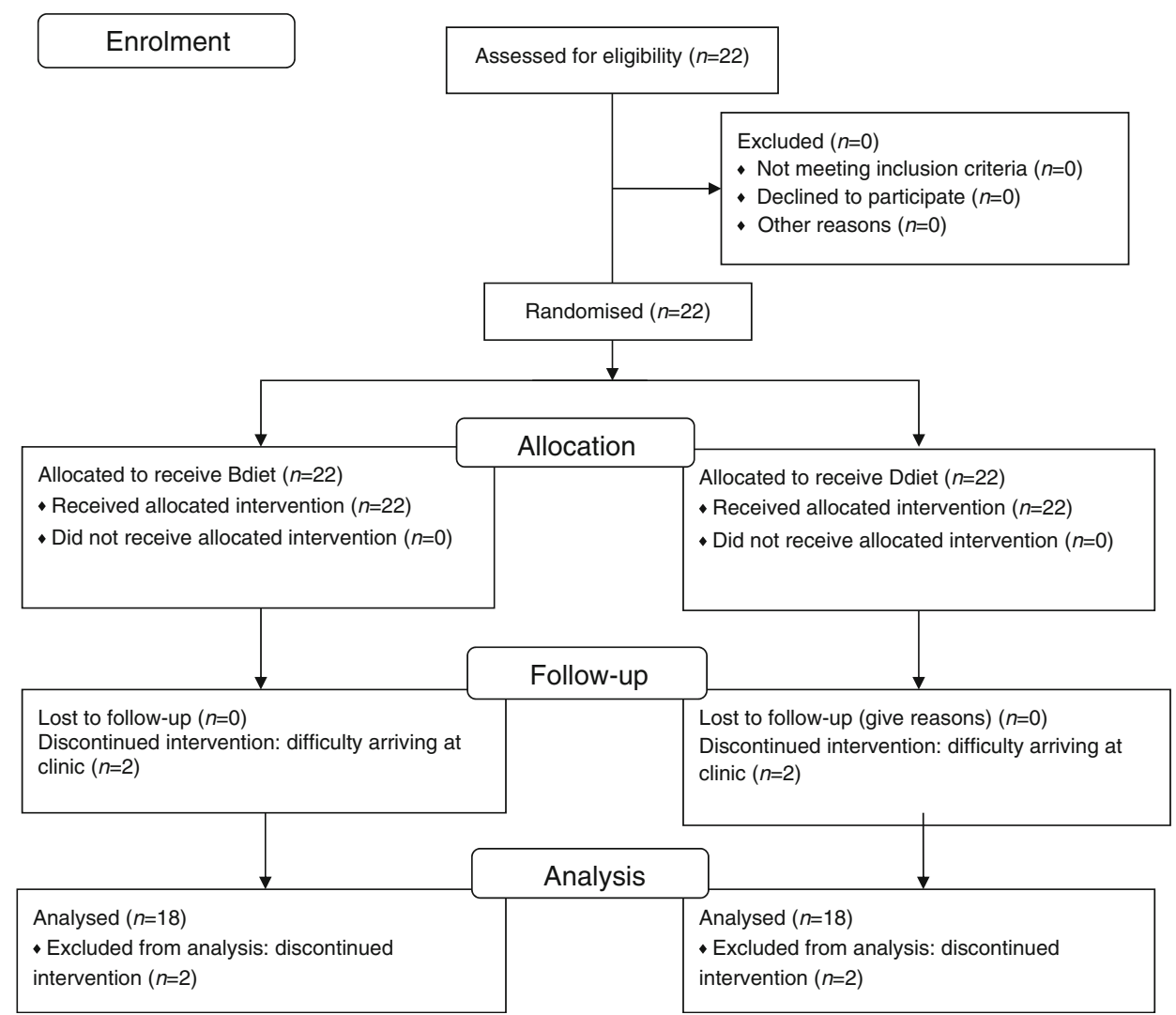


a

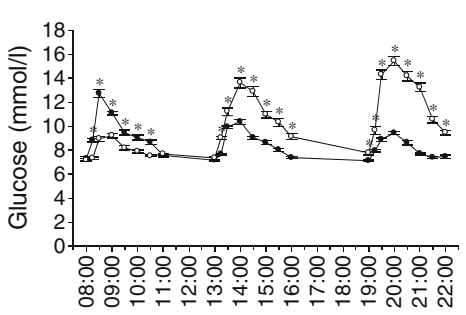

C

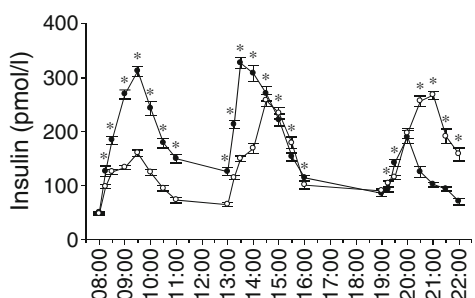

e

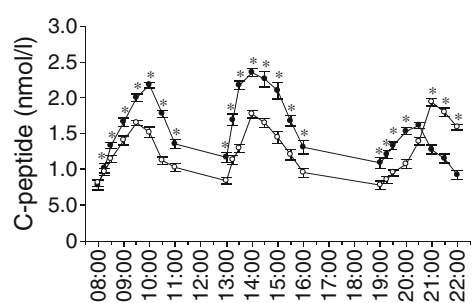

g

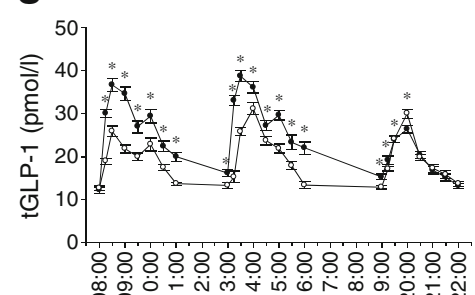

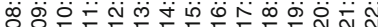

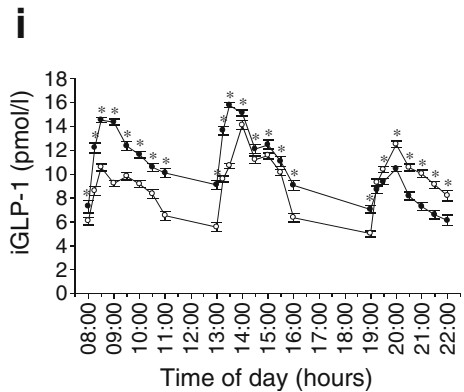

Fig. 2 All-day graphs for glucose, insulin, C-peptide, tGLP-1 and iGLP1. White circles, Ddiet group; black circles, Bdiet group. ${ }^{*} p<0.05$

$\mathrm{AUC}_{\text {insulin, }}, \mathrm{AUC}_{\mathrm{C} \text {-peptide }}$ and $\mathrm{AUC}_{\mathrm{tGLP}-1}$ after breakfast, lunch and dinner were higher by $20 \%$ during the Bdiet than during the Ddiet $\left(p<0.001, t\right.$ test). Integrated $\mathrm{AUC}_{\mathrm{iGLP}-1}$ after breakfast, lunch and dinner was higher by $10 \%$ during the Bdiet than during the Ddiet $(p<0.001, t$ test).
Plasma glucose and hormonal profiles after high-energy breakfast vs high-energy dinner After high-energy breakfast, glucose $\mathrm{AUC}_{0-180 \mathrm{~min}}$ and its peak were reduced by $24 \%$ compared with the glucose response after high-energy dinner ( $p<0.001, t$ test) (Table 3, Fig. 2). During the early interval, $\mathrm{AUC}_{0-30 \mathrm{~min}}$, plasma glucose was lower by $10 \%$ after highenergy breakfast vs high-energy dinner $(p<0.006, t$ test). After reaching their peak, plasma glucose levels decreased rapidly after the high-energy breakfast whereas after the high-energy dinner, plasma glucose persisted and remained significantly higher.

The insulin response to the meal challenge was more rapid and higher after high-energy breakfast compared with highenergy dinner (Table 3, Fig. 2). Insulin $\mathrm{AUC}_{0-180 \min }$ was $11 \%$ higher after high-energy breakfast than after high-energy dinner $(p<0.001, t$ test) (Table 3$)$. During the first 30 min, plasma insulin levels increased more rapidly after high-energy breakfast than after high-energy dinner $(p<0.008, t$ test). During the late interval, $\mathrm{AUC}_{60-180 \mathrm{~min}}$, insulin secretion did not differ $(p<0.11)$ between the meals. After high-energy breakfast, peak insulin secretion occurred at $60 \mathrm{~min}, 12 \%$ higher $(p<0.002, t$ test) than after high-energy dinner. After highenergy dinner, peak insulin secretion occurred at $120 \mathrm{~min}$. After meal ingestion, patterns of C-peptide levels mirrored those of insulin with a more rapid and higher increase after high-energy breakfast. High-energy breakfast resulted in a higher C-peptide early response and $\mathrm{AUC}_{0-180 \min }$, by $17 \%$ ( $p<0.001, t$ test) compared with high-energy dinner (Table 3 ).

The $\mathrm{AUC}_{0-180 \mathrm{~min}}$ for tGLP-1 was $29 \%$ higher after highenergy breakfast than after high-energy dinner $(p<0.001$, $t$ test) (Table 3, Fig. 2). Plasma tGLP-1 increased rapidly after high-energy breakfast and peaked at $30 \mathrm{~min}$. In contrast, after high-energy dinner, the increase in plasma tGLP-1 was more gradual and peaked at $60 \mathrm{~min}$. Compared with high-energy dinner, the high-energy breakfast resulted in $21 \%$ higher tGLP-1 levels ( $p<0.001$, post hoc $t$ test). The high-energy breakfast elicited $30-35 \%$ higher early and late interval for tGLP-1 ( $p<0.001, t$ test). Similarly, iGLP-1 plasma levels peaked at $30 \mathrm{~min}$ after high-energy breakfast compared with a peak at $60 \mathrm{~min}$ after high-energy dinner. The peak level of iGLP-1 was higher by $16 \%$ after high-energy breakfast than after high-energy dinner $(p<0.001$, post hoc $t$ test $)$. The $\mathrm{AUC}_{0-180 \mathrm{~min}}$, as well as the early and late intervals for tGLP-1 and iGLP-1, were 14-35\% higher after high-energy breakfast than after high-energy dinner $(p<0.001, t$ test $)$.

Plasma glucose and hormonal profiles after Bdiet lunch vs Ddiet lunch Glucose $\mathrm{AUC}_{0-180 \mathrm{~min}}$ and its peak were lower by $21-25 \%$ after Bdiet lunch vs Ddiet lunch ( $p<0.001, t$ test) (Table 3, Fig. 2). The glucose early and late interval was lower by $13 \%$ and $24 \%$, respectively, after Bdiet lunch than after Ddiet lunch $\left(p<0.006, t\right.$ test). Insulin $\mathrm{AUC}_{0-180 \mathrm{~min}}$ was higher by $23 \%$ after Bdiet lunch than after Ddiet lunch. In addition, 
Table 3 AUC at different time intervals of breakfast vs dinner and HE lunch of Bdiet vs Ddiet

\begin{tabular}{|c|c|c|c|c|c|c|c|c|}
\hline AUC & Bdiet HE breakfast & Ddiet HE dinner & $p$ value & $\%$ change & Bdiet lunch & Ddiet lunch & $p$ value & $\%$ change \\
\hline \multicolumn{9}{|c|}{ Glucose $(\mathrm{mmol} / 1 \times \min )$} \\
\hline $0-30 \mathrm{~min}$ & $283 \pm 16$ & $311 \pm 27$ & $<0.006$ & -10 & $243 \pm 6$ & $275 \pm 16$ & $<0.001$ & -13 \\
\hline $60-180 \mathrm{~min}$ & $1,094 \pm 56$ & $1,513 \pm 125$ & $<0.001$ & -27 & $1,038 \pm 55$ & $1,368 \pm 124$ & $<0.001$ & -24 \\
\hline $0-180 \mathrm{~min}$ & $1,735 \pm 76$ & $2,270 \pm 167$ & $<0.001$ & -24 & $1,586 \pm 9$ & $2,015 \pm 67$ & $<0.001$ & -21 \\
\hline \multicolumn{9}{|c|}{ Insulin (pmol/1× min) } \\
\hline $0-30 \mathrm{~min}$ & $3,653 \pm 757$ & $3,104 \pm 326$ & $<0.008$ & 19 & $6,587 \pm 590$ & $3,327 \pm 382$ & $<0.001$ & 49 \\
\hline $60-180 \mathrm{~min}$ & $28,308 \pm 2,952$ & $26,780 \pm 3,702$ & 0.111 & 8 & $25,683 \pm 3,841$ & $24,155 \pm 3,118$ & 0.140 & 8 \\
\hline $0-180 \mathrm{~min}$ & $38,753 \pm 3,695$ & $34,572 \pm 4,257$ & $<0.001$ & 11 & $41,781 \pm 4,424$ & $32,260 \pm 3,792$ & $<0.001$ & 23 \\
\hline \multicolumn{9}{|c|}{ C-peptide $(\mathrm{nmol} / 1 \times \min )$} \\
\hline $0-30 \mathrm{~min}$ & $31 \pm 7$ & $26 \pm 5$ & 0.042 & 17 & $50 \pm 7$ & $33 \pm 5$ & $<0.001$ & 35 \\
\hline $60-180 \mathrm{~min}$ & $224 \pm 19$ & $194 \pm 15$ & $<0.001$ & 16 & $236 \pm 36$ & $170 \pm 25$ & $<0.001$ & 28 \\
\hline $0-180 \mathrm{~min}$ & $300 \pm 29$ & $250 \pm 20$ & $<0.001$ & 17 & $354 \pm 43$ & $249 \pm 23$ & $<0.001$ & 30 \\
\hline \multicolumn{9}{|c|}{ tGLP-1 (pmol/1× min) } \\
\hline $0-30 \mathrm{~min}$ & $817 \pm 104$ & $533 \pm 51$ & $<0.001$ & 35 & $906.1 \pm 89$ & $519.7 \pm 115$ & $<0.001$ & 43 \\
\hline $60-180 \mathrm{~min}$ & $3,178 \pm 388$ & $2,238 \pm 241$ & $<0.001$ & 30 & $3,271 \pm 453$ & $2,566 \pm 352$ & $<0.001$ & 22 \\
\hline $0-180 \mathrm{~min}$ & $5,063 \pm 489$ & $3,581 \pm 256$ & $<0.001$ & 29 & $5,298 \pm 523$ & $3,938 \pm 490$ & $<0.001$ & 26 \\
\hline \multicolumn{9}{|c|}{ iGLP-1 (pmol/1× min) } \\
\hline $0-30 \mathrm{~min}$ & $347 \pm 36$ & $255 \pm 27$ & $<0.001$ & 27 & $391.3 \pm 26$ & $265.5 \pm 22$ & $<0.001$ & 32 \\
\hline $60-180 \mathrm{~min}$ & $1,403 \pm 124$ & $1,202 \pm 133$ & $<0.001$ & 14 & $1,431 \pm 161$ & $1,294 \pm 104$ & $<0.002$ & 11 \\
\hline $0-180 \mathrm{~min}$ & $2,183 \pm 154$ & $1,799 \pm 161$ & $<0.001$ & 18 & $2,286 \pm 64$ & $1,931 \pm 113$ & $<0.001$ & 16 \\
\hline
\end{tabular}

the early prandial insulin $\mathrm{AUC}_{0-30 \text { min }}$ was higher by almost $50 \%$ after Bdiet lunch vs Ddiet lunch ( $p<0.001, t$ test). Insulin reached peak levels $30 \mathrm{~min}$ after the Bdiet lunch and $90 \mathrm{~min}$ after the Ddiet lunch, with the Bdiet lunch producing higher peak levels ( $p<0.001, t$ test) (Table 3, Fig. 2). Throughout the 180 min of analysis, C-peptide levels were $30 \%$ higher after the Bdiet lunch than after the Ddiet lunch $(p<0.001, t$ test $)$ and tGLP-1 and iGLP-1 levels were 16-26\% higher after the Bdiet lunch than after the Ddiet lunch $(p<0.001, t$ test). In addition, tGLP-1 and iGLP-1 peaked 30 min earlier after Bdiet lunch compared with Ddiet lunch.

\section{Discussion}

In this study, we demonstrate in individuals with type 2 diabetes that a meal schedule of high-energy breakfast and lowenergy dinner (Bdiet) leads to overall increased GLP-1 and insulin levels and to reduced hyperglycaemia throughout the day compared with a reverse meal schedule (Ddiet). Although both diets had an identical day-long energy composition, the difference in timing led to a significant reduction in overall glucose excursions in the Bdiet compared with the Ddiet. In parallel, the integrated AUCs for insulin, C-peptide, iGLP-1 and tGLP-1 secretion were significantly enhanced along the entire day in the Bdiet compared with the Ddiet schedule. These observations suggest that a change in meal timing influences the overall daily rhythm of postprandial insulin, incretin and glycaemic excursions. Our findings showing a significantly reduced overall glycaemia with the Bdiet schedule are consistent with studies in animal models. Impaired peripheral clock gene expression as a result of breakfast skipping or reduced food intake in the first meal of the day along with high-energy dinner, has been associated with increased lipogenesis and higher overall daily glucose excursions, despite no differences in daily total or fat-derived energy [12, 22]. Alternatively, a specific change in the meal timing (i.e. increasing breakfast and reducing dinner energy content) may also restore clock gene expression in obese and diabetic animals, resulting in reduced plasma glucose and triacylglycerol levels and body weight $[12,22]$. Recently, it was reported that high-energy breakfast with reduced dinner improved insulin sensitivity and decreased body weight, glucose excursions and $\mathrm{HbA}_{1 \mathrm{c}}$ among obese and diabetic patients [24-27]. The omission of breakfast was also associated with increased risk of type 2 diabetes, poor glycaemic control, higher $\mathrm{HbA}_{1 \mathrm{c}}$, increased lipogenesis, visceral adiposity and high blood pressure and increased cardiovascular risk despite the same daily energy intake in individuals with type 2 diabetes [20, 21]. Although this study investigated the acute effect of meals, long-term interventions should determine whether adhering to this diet would successfully lower $\mathrm{HbA}_{1 \mathrm{c}}$ levels. This issue was addressed in our previous study, in which eating a large breakfast each day for 3 months led to a $5 \%$ reduction in 
$\mathrm{HbA}_{1 \mathrm{c}}$ levels [27]. Thus, meal timing schedule may be a crucial factor in the improvement of glucose balance and prevention of complications in type 2 diabetes and lends further support to the role of the circadian system in metabolic regulation.

The postprandial glucose response after the high-energy breakfast was significantly lower than after the low-energy breakfast, and decreased rapidly after the peak at $30 \mathrm{~min}$. In contrast, the plasma glucose after high-energy dinner peaked higher than after the low-energy dinner, and remained elevated until the end of the postprandial response. Decline in carbohydrate tolerance toward the evening, with more prolonged and higher postprandial glycaemic response to identical meals in the evening than in the morning has been shown in healthy individuals and those with type 2 diabetes [4-8]. Notably, the $2 \mathrm{~h}$ post-meal glycaemia was significantly lower after the high-energy breakfast than after the high-energy dinner, and was below the upper limit $(10 \mathrm{mmol} / \mathrm{l})$ of $2 \mathrm{~h}$ post-meal glycaemia recommended in the recent ADA guidelines for the prevention of cardiovascular events in patients with type 2 diabetes. In parallel, significantly higher insulin and C-peptide responses were observed after the high-energy breakfast than after the high-energy dinner. This greater and more rapid early prandial insulin secretion after the highenergy breakfast has further importance since in type 2 diabetes the deficiency of early prandial insulin is the major contributor to postprandial hyperglycaemia and cardiovascular risk $[2,3]$. The mechanism of better glucose tolerance after high-energy breakfast than after an identical dinner may be in part the result of clock regulation that triggers higher beta cell responsiveness in the morning, lower hepatic insulin extraction after breakfast than after dinner [7] and the increase in insulin-mediated muscle glucose uptake in the morning [7, 10]. Thus, the assignment of major energy load at breakfast, when beta cell responsiveness and insulin-mediated muscle glucose uptake are at optimal levels, seems an adequate strategy to decrease PPHG in patients with type 2 diabetes. The contribution to our results made by different clearance of insulin cannot be excluded, although ratios of insulin to C-peptide (a rough estimate of hepatic extraction) did not differ between the diets.

The early prandial increase in GLP-1 during the first $30 \mathrm{~min}$ after high-energy breakfast coincided with the early prandial insulin response. These results are supported by previous findings in which AUC30 $0_{\mathrm{iGLP}-1}$ correlated significantly with AUC30 $0_{\text {insulin }}[6]$. This suggests that the more rapid and higher early insulin response after the high-energy breakfast may be a result of a more rapid early prandial GLP-1 level, which mediates the potentiation of beta cell function. Recently, the circadian rhythm of GLP-1 secretory responses was shown in animals, with increased release after the first meal at the beginning of the normal feeding period and this profile was correlated with insulin secretion [9]. Moreover, when this first meal was omitted, both GLP-1 and insulin rhythms were completely inverted along with disruption of clock gene expression. Collectively, these results indicate that the peripheral clock in intestinal $\mathrm{L}$ cells, which drives the circadian expression of GLP-1, can be modulated by different meal timing.

Glucose excursions were reduced after lunch preceded by high-energy breakfast (Bdiet) compared with after an identical lunch preceded by low-energy breakfast (Ddiet). This reduced glucose response was associated with rapid and significantly enhanced insulin, tGLP-1 and iGLP-1 responses. This secondmeal phenomenon suggests that by changing meal timing schedule, it is possible to enhance the incretin and insulinotropic effects, achieving significant reduction in PPHG through the day in patients with type 2 diabetes. Several theories have been suggested to explain the second-meal phenomenon in type 2 diabetes, including differences in the amount and the pattern of insulin secretion, augmentation of beta cell function and increased incretin response [26, 28]. The high GLP-1 response to lunch after high-energy breakfast suggests that incretin hormones may play an important role in the second-meal phenomenon. Indeed, this phenomenon was absent after a repeated intravenous glucose tolerance test [29], but not after a meal tolerance test $[26,30,31]$.

One limitation of our study is that the macronutrient composition varied according to the time of day. Thus, some of the results may also be due to the timing of macronutrient ingestion. Another limitation is that a group of healthy individuals was not included. Although we show the effect of two different meal timing schedules on the overall hyperglycaemia and on the potentiation of insulin and incretin secretion throughout the day and the effect of second-meal phenomenon in diabetic individuals, we cannot determine whether these effects also occur in healthy people. In addition, insulin sensitivity, suppression of endogenous glucose production and gastric emptying were not examined.

In conclusion, the results of this study support a role for diurnal regulation in glycaemic control in patients with type 2 diabetes. We demonstrated that a larger percentage of daily energy consumed at breakfast is associated with significant reduction in overall PPHG in patients with type 2 diabetes. The findings also suggest the possibility of applying the second-meal phenomenon as a way of reducing postprandial glucose excursion, especially at lunch. This, along with the benefits of the Bdiet schedule in reducing overall hyperglycaemia, may have a therapeutic advantage for the achievement of optimal metabolic control and may prevent cardiovascular and other complications of type 2 diabetes.

Acknowledgement The authors would like to thank M. Barnea (The Weizmann Institute of Science, Rehovot, Israel) for statistical assistance. 
Duality of interest The authors declare that there is no duality of interest associated with this manuscript.

Contribution statement JW contributed to the conception and design of the study, acquired and interpreted data and drafted the article. OF and DJ contributed to the conception and design of the study, acquired, analysed and interpreted data and drafted and revised the article. BA researched data, contributed to the interpretation of the data and drafted and revised the article. HRR, YB and ZL contributed to the conception and design of the study, acquired and interpreted data, organised the randomisation and drafted the article. All listed authors approved the final version of the manuscript. DJ is the guarantor of this work.

\section{References}

1. Monnier L, Lapinski H, Colette C (2003) Contributions of fasting and postprandial plasma glucose increments to the overall diurnal hyperglycemia of type 2 diabetic patients: variations with increasing levels of $\mathrm{HbA}(1 \mathrm{c})$. Diabetes Care 26:881-885

2. Ceriello A, Colagiuri S, Gerich J, Tuomilehto J, Guideline Development G (2008) Guideline for management of postmeal glucose. Nutrition, metabolism, and cardiovascular diseases. Nutr Metab Cardiovasc Dis 18:S17-S33

3. Szuszkiewicz-Garcia MM, Davidson JA (2014) Cardiovascular disease in diabetes mellitus: risk factors and medical therapy. Endocrinol Metab Clin N Am 43:25-40

4. Morgan LM, Shi JW, Hampton SM, Frost G (2012) Effect of meal timing and glycaemic index on glucose control and insulin secretion in healthy volunteers. Br J Nutr 108:1286-1291

5. van Cauter E, Shapiro ET, Tillil H, Polonsky KS (1992) Circadian modulation of glucose and insulin responses to meals: relationship to cortisol rhythm. Am J Physiol 262:E467-E475

6. Lindgren O, Mari A, Deacon CF et al (2009) Differential islet and incretin hormone responses in morning versus afternoon after standardized meal in healthy men. J Clin Endocrinol Metab 94:28872892

7. Saad A, Dalla Man C, Nandy DK et al (2012) Diurnal pattern to insulin secretion and insulin action in healthy individuals. Diabetes 61:2691-2700

8. Gibbs M, Harrington D, Starkey S, Williams P, Hampton S (2014) Diurnal postprandial responses to low and high glycaemic index mixed meals. Clin Nutr 33:889-894

9. Gil-Lozano M, Mingomataj EL, Wu WK, Ridout SA, Brubaker PL (2014) Circadian secretion of the intestinal hormone GLP-1 by the rodent L cell. Diabetes 63:3674-3685

10. Dyar KA, Ciciliot S, Wright LE et al (2014) Muscle insulin sensitivity and glucose metabolism are controlled by the intrinsic muscle clock. Mol Metab 3:29-41

11. Froy O (2010) Metabolism and circadian rhythms-implications for obesity. Endocr Rev 31:1-24

12. Wu T, Sun L, ZhuGe F et al (2011) Differential roles of breakfast and supper in rats of a daily three-meal schedule upon circadian regulation and physiology. Chronobiol Int 28:890-903

13. Prasai MJ, Mughal RS, Wheatcroft SB, Kearney MT, Grant PJ, Scott EM (2013) Diurnal variation in vascular and metabolic function in diet-induced obesity: divergence of insulin resistance and loss of clock rhythm. Diabetes 62:1981-1989
14. Yoshino J, Imai S (2010) A clock ticks in pancreatic beta cells. Cell Metab 12:107-108

15. Sadacca LA, Lamia KA, deLemos AS, Blum B, Weitz CJ (2011) An intrinsic circadian clock of the pancreas is required for normal insulin release and glucose homeostasis in mice. Diabetologia 54:120-124

16. Reppert SM, Weaver DR (2002) Coordination of circadian timing in mammals. Nature 418:935-941

17. Damiola F, Le Minh N, Preitner N, Kornmann B, Fleury-Olela F, Schibler U (2000) Restricted feeding uncouples circadian oscillators in peripheral tissues from the central pacemaker in the suprachiasmatic nucleus. Genes Dev 14:2950-2961

18. Hara R, Wan K, Wakamatsu H et al (2001) Restricted feeding entrains liver clock without participation of the suprachiasmatic nucleus. Genes Cells 6:269-278

19. Loboda A, Kraft WK, Fine B et al (2009) Diurnal variation of the human adipose transcriptome and the link to metabolic disease. BMC Med Genomics 2:7

20. Reutrakul S, Hood MM, Crowley SJ, Morgan MK, Teodori M, Knutson KL (2014) The relationship between breakfast skipping, chronotype, and glycemic control in type 2 diabetes. Chronobiol Int 31:64-71

21. Mekary RA, Giovannucci E, Willett WC, van Dam RM, Hu FB (2012) Eating patterns and type 2 diabetes risk in men: breakfast omission, eating frequency, and snacking. Am J Clin Nutr 95:1182-1189

22. Fuse Y, Hirao A, Kuroda H, Otsuka M, Tahara Y, Shibata S (2012) Differential roles of breakfast only (one meal per day) and a bigger breakfast with a small dinner (two meals per day) in mice fed a high-fat diet with regard to induced obesity and lipid metabolism. J Circadian Rhythms 10:4

23. Sherman H, Genzer Y, Cohen R, Chapnik N, Madar Z, Froy O (2012) Timed high-fat diet resets circadian metabolism and prevents obesity. FASEB J 26:3493-3502

24. Jakubowicz D, Froy O, Wainstein J, Boaz M (2012) Meal timing and composition influence ghrelin levels, appetite scores and weight loss maintenance in overweight and obese adults. Steroids 77:323-331

25. Jakubowicz D, Barnea M, Wainstein J, Froy O (2013) High caloric intake at breakfast vs. dinner differentially influences weight loss of overweight and obese women. Obesity 21: 2504-2512

26. Jakubowicz D, Froy O, Ahrén B et al (2014) Incretin, insulinotropic and glucose-lowering effects of whey protein pre-load in type 2 diabetes: a randomised clinical trial. Diabetologia 57:1807-1811

27. Rabinovitz HR, Boaz M, Ganz T et al (2014) Big breakfast rich in protein and fat improves glycemic control in type 2 diabetics. Obesity 22:E46-E54

28. Lee SH, Tura A, Mari A et al (2011) Potentiation of the early-phase insulin response by a prior meal contributes to the second-meal phenomenon in type 2 diabetes. Am J Physiol Endocrinol Metab 301: E984-E990

29. Ravanam A, Jeffery J, Nehlawi M, Abraira C (1991) Improvement of glucose-primed intravenous glucose tolerance and correction of acute insulin decrement by glipizide in type II diabetes. Metab Clin Exp 40: $1173-1177$

30. Jovanovic A, Gerrard J, Taylor R (2009) The second-meal phenomenon in type 2 diabetes. Diabetes Care 32:1199-1201

31. Bonuccelli S, Muscelli E, Gastaldelli A et al (2009) Improved tolerance to sequential glucose loading (Staub-Traugott effect): size and mechanisms. Am J Physiol Endocrinol Metab 297: E532-E537 